\title{
Increased expression of Fc $\gamma$ receptors on neutrophils and monocytes may reflect ongoing bacterial infection
}

\author{
C. M. HERRA, C. T. KEANE and A. WHELAN* \\ Department of Clinical Microbiology, Sir Patrick Dun Research Laboratory and *Department of Immunology, \\ Central Pathology Laboratory, St James's Hospital, Dublin 8, Republic of Ireland
}

\begin{abstract}
The Fc $\gamma$ R receptors for IgG, FcyRI, Fc $\gamma$ RII and Fc $\gamma$ RIII were measured on neutrophils and monocytes from 36 patients suspected of systemic infection. These results were compared with 30 blood donor controls to assess the level of expression as an early indicator of bacterial infection. FcrRI expression on neutrophils was found to be significantly increased from patients with systemic or localised infections, when compared to the non-infected patient group, i.e., patients with no cultural evidence of bacterial infections, $(p=0.02, p=0.04)$ or normal controls $(p<0.0001, p=0.0005)$. Fc $\gamma R I$ expression on monocytes was also significantly increased in both of the infected groups compared to normal controls $(p<0.0001, p=0.001$ ); however, no significant difference could be seen when compared with the non-infected patients. Fc $\gamma$ RIII was found to be significantly increased on a subset of monocytes in patients with systemic or localised infections compared to the non-infected group $(p=0.009, p=0.006)$ and compared to the normal controls $(p=0.009, p=0.003)$. Infections caused by gramnegative bacilli induced a higher FcyR response than infection with either streptococci or staphylococci. These data suggest that the measurement of FcyRI on neutrophils and Fc $\gamma$ RIII on monocytes may be a useful rapid indicator of bacterial infection.
\end{abstract}

\section{Introduction}

Despite appropriate antimicrobial chemotherapy, one of the most frequent and serious problems facing clinicians is the management of patients with severe infection [1,2]. Prompt diagnosis is essential to improve prognosis. The development of methods for the early detection of infection based on the measurement of host immune response, rather than sometimes lengthy culture techniques, would be advantageous.

Human phagocytes express three distinct classes of receptors for the $\mathrm{Fc}$ domain of IgG designated $\mathrm{Fc} \gamma \mathrm{RI}$ (CD64), Fc $\gamma$ RII (CD32) and Fc $\gamma$ RIII (CD16) [3, 4]. In the body, these receptors form a two-way bridge between the humoral response and the cellular branch of the immune system [5]. When $\mathrm{Fc} \gamma$ receptors $(\mathrm{Fc} \gamma \mathrm{R})$ interact with immunoglobulin, various important biological responses are triggered, such as phagocytosis, endocytosis, antibody-dependent cellular cytotoxicity, release of inflammatory mediators and enhancement of antigen presentation [6]. Therefore, it is expected that as these receptors and their regulation have a priority in infection, the cellular immune response to bacterial challenge would involve in-

Received 30 May 1995; accepted 18 Aug. 1995.

Corresponding author: C. M. Herra. creased expression of Fc $\gamma \mathrm{R}$ on the phagocytic cells, at an early stage of infection.

Early studies have already indicated that Fc $\gamma \mathrm{RI}$ expression is increased in acute bacterial infection [7]. Other studies have shown that IFN- $\gamma$ can bring about a dramatic increase in Fc $\gamma \mathrm{RI}$ expression on both monocytes [8] and neutrophils [9]. More recent work has also shown that Fc $\gamma \mathrm{RI}$ expression is increased on neutrophils and augmented on monocytes in patients with localised $\beta$-haemolytic streptococcal (potent IFN- $\gamma$ inducers) pharyngitis [10]. This study was designed to investigate whether $\mathrm{Fc} \gamma \mathrm{R}$ expression is also increased in systemic infection and, if so, whether its measurement could be used as an early indicator of infection.

\section{Materials and methods}

Patient population

Whole blood EDTA samples were collected from 36 patients with suspected systemic infection, at the time of blood culture analysis. Thirty EDTA samples were also collected from blood donors as healthy controls. Of the 36 patients tested, 16 had systemic infection, i.e., clinically significant bacteria were isolated from the blood culture. These isolates consisted of the 
following: Staphylococcus aureus $(\mathrm{n}=5$, four of which were methicillin- and gentamicin-resistant strains); Streptococcus pneumoniae $(\mathrm{n}=2)$, Enterococcus $\mathrm{sp}$. $(\mathrm{n}=1)$, Escherichia coli $(\mathrm{n}=5)$, Enterobacter cloacae $(\mathrm{n}=1)$, Klebsiella oxytoca $(\mathrm{n}=1)$ and Pseudomonas putida $(\mathrm{n}=1)$. Six patients were blood-culture negative but had severe localised bacterial infections at other sites. Of these patients, four had infections in the lower respiratory tract caused by gram-negative pathogens such as $K$. pneumoniae, $P$. aeruginosa, $E$. coli and Haemophilus influenzae. Another of these patients had peritonitis due to $E$. coli whilst the remaining patient had Clostridium difficile-associated diarrhoea. The remaining 14 patients tested had no cultural evidence of bacterial infection. Viral studies were not performed on the patients tested.

\section{Fluorescent staining}

Fc $\gamma \mathrm{R}$ expression was detected by a direct staining immunofluorescence technique with fluorescein isothiocyanate (FITC)-labelled monoclonal antibodies (MAbs) against Fc $\gamma$ RI (CD64), Fc $\gamma$ RII (CD32) and FcyRIII (CD16) (Medarex, Princeton, NJ, USA). Phycoerythrin (PE)-labelled CD14 (BD) was used as a monocytic marker. A non-sense antibody, $\gamma_{1} \gamma_{2}$, (BD) was also used as a control for non-specific binding. The receptors were stained by a whole blood lysis technique on a single EDTA specimen which was divided into four $100-\mu \mathrm{l}$ volumes. Five $\mu \mathrm{l}$ of the three Fc $\gamma \mathrm{R}$ antibodies were added to three of the tubes, respectively. Non-sense antibody $(5 \mu \mathrm{l})$ was added to the fourth tube. A further $5 \mu$ l of the CD14 antibody was added to each tube. After incubation for $10 \mathrm{~min}$ at room temperature, $2 \mathrm{ml}$ of red cell lysis solution (BD) were added to each tube and tubes were incubated for a further $10 \mathrm{~min}$. Tubes were centrifuged at $5000 \mathrm{rpm}$ for $5 \mathrm{~min}$ after which the supernate was discarded. Samples were then washed in FACS flow fluid (BD), and fixed in paraformaldehyde $1 \%$ in PBS.

\section{Flow cytometric analysis}

Flow cytometric measurement of the stained samples was performed with a single laser (Argon ion $488 \mathrm{~nm}$ ) FACSCAN flow cytometer (BD). CaliBRITE beads (BD) were used daily to set the PMT voltages and the fluorescent compensation and also as a check on instrument sensitivity. Patient samples were acquired at a rate of c. 200 cells/s, such that 10000 events were counted on each sample. LYSIS software was then used to estimate MAb binding on the neutrophils and the monocytes. Neutrophils were distinguished and electronically gated on the basis of their size and granularity, by means of a dot plot of forward scatter (FSC) versus side scatter (SSC). Gating on the PE-labelled monocytes was performed with a dot plot of the fluorescent intensity of FITC (FL1) versus the fluorescence intensity of PE (FL2). The mean fluorescence intensity (MFI) of each of the cell populations was then calculated from a profile histogram of the FLl for each sample. For Fc $\gamma$ RIII, which is expressed only on a subpopulation of monocytes, the percentage of monocytes expressing this receptor was also calculated from the FL1 histogram. A marker was set on the subpopulation of monocytes with increased Fc $\gamma$ RIII expression, against a background control, and the percentage of cells within the marked region was then calculated with the 'Histats' in the LYSIS programme. The percentage of monocytes with increased expression of FcyRIII was calculated for each of the patient samples, but on a smaller number of controls $(n=15)$ than originally tested.

\section{Statistical analysis}

Mean MFI values for the three receptors, on both neutrophils and monocytes, were calculated for each of the patient groups and the controls. MFI values for the patient groups and the controls were also compared by analysis of variance (ANOVA) and a modified $t$ test for unpaired samples, based on a pooled estimate of variance. A p value $<0.05$ was taken to be significant. Similiar calculations, including patient group mean values and $t$ test comparisons, were performed on the percentage of monocytes with increased expression of Fc $\gamma$ RIII.

\section{Results}

Examination of MFI values indicated that whilst Fc $\gamma \mathrm{R}$ expression was increased in patients with either systemic or localised infection, there was little difference in expression between the non-infected patients and the controls (Table 1). There was no significant difference in the level of expression of Fc $\gamma R I$, Fc $\gamma$ RII or Fc $\gamma$ RIII in patients with either systemic or localised infection. However, for Fc $\gamma \mathrm{RI}$ expression, there was a significant difference between patients with either systemic or localised infection, compared to the controls, for both neutrophils $(\mathrm{p}<0.0001, \mathrm{p}=0.0005)$ (Fig. 1A) and monocytes $(\mathrm{p}<0.0001, \mathrm{p}=0.001)$ (Fig. 1B). Fc $\gamma \mathrm{RI}$ expression was also significantly increased in the two infected patient groups compared to patients without evidence of infection, for neutrophils $(p=0.02, p=0.04)$ (Fig. 1A). The monocytic expression of Fc $\gamma$ RIII was also significantly increased in patients with either systemic or localised infection compared to controls $(\mathrm{p}<0.0001, \mathrm{p}<0.0001)$. This increased expression of Fc $\gamma$ RIII in infection was more apparent when comparisons were made by calculations involving the percentage of monocytes expressing increased levels of this receptor. In this case the percentage of monocytes showing a higher level of FcyRIII expression was significantly increased in both infected groups compared not only to the controls $(p=0.009, p=0.003)$, but also to patients without evidence of infection $(p=0.009, p=0.006)$ (Fig. 1C). 
Table 1. Mean fluorescence intensity (MFI) values for Fc $\gamma$ receptor expression on neutrophils and monocytes in patient and control groups.

\begin{tabular}{|c|c|c|c|c|c|c|}
\hline \multirow{3}{*}{$\begin{array}{l}\text { Patient } \\
\text { groups* }\end{array}$} & \multicolumn{6}{|c|}{ Mean and range ( $\pm 1 \mathrm{SD})$ MFI values } \\
\hline & \multicolumn{3}{|c|}{ Neutrophils } & \multicolumn{3}{|c|}{ Monocytes } \\
\hline & $\mathrm{Fc} \gamma \mathrm{RI}$ & Fc $\gamma$ RII & Fc $\gamma$ RIII & Fc $\gamma R I$ & Fc $\gamma$ RII & Fc $\gamma$ RIII \\
\hline Control & $26(21-31)$ & $120(105-135)$ & $491(324-658)$ & $75(64-86)$ & $104 \quad(87-121)$ & $29(16-42)$ \\
\hline Non-infected & $32(24-40)$ & $147(109-181)$ & $443(283-603)$ & $115(82-148)$ & $159(123-195)$ & $48(34-62)$ \\
\hline Systemic infection & $62(34-90)$ & $147(103-191)$ & $443(178-708)$ & $156(95-217)$ & $127(82-172)$ & $78(18-138)$ \\
\hline Localised infection & $76(43-109)$ & $161(130-192)$ & $673(249-1097)$ & $199(76-322)$ & $151 \quad(91-211)$ & $95(46-144)$ \\
\hline
\end{tabular}

*Controls: blood donors; non-infected patients: patients without cultural evidence of bacterial infection; systemic infection: patients with systemic infection; localised infection: patients with negative blood culture but significant infection at localised sites.

Analysis of the MFI values obtained in patients with systemic infection also indicated that some bacterial isolates induced a higher $F c \gamma R$ response than others. For Fc $\gamma \mathrm{RI}$, on both neutrophils and monocytes, gramnegative bacilli appeared to elicit a higher receptor response than either streptococci or staphylococci (Fig. 2A). Similarly, for Fc $\gamma$ RIII, the percentage of monocytes with increased expression of this receptor, showed a notable increase in infections caused by gram-negative bacilli (Fig. 2B). In turn, for both Fc $\gamma$ RI and Fc $\gamma$ RIII, Streptococcus spp. such as $S$. pneumoniae, induced a higher $\mathrm{Fc} \gamma \mathrm{R}$ response than most of the staphylococcal isolates. The gram-negative organisms involved in localised infections also induced a high $\mathrm{Fc} \gamma \mathrm{R}$ response, but at a lower level than the gram-negative organisms causing systemic infection.

\section{Discussion}

It is now recognised that the high mortality rate associated with systemic infection arises not only from the presence and establishment of organisms in the bloodstream, but also from the body's own immune response to the infection, a response that can trigger a potentially fatal cascade of inflammatory mediators $[1,11,12]$. Terms such as sepsis, sepsis syndrome and septic shock are no longer used to denote separate infectious disorders. These terms are now used to represent increasingly severe stages of the same infectious process, the progress of which relates to the severity of the systemic response rather than to the infection itself [13].

The development of suitable antimicrobial therapy has brought about a great reduction in the mortalities associated with systemic infection. However, administration of effective antimicrobial agents can have many contraindications for the patient. In gramnegative infection, for example, septic shock can be triggered as a primary response to the release of lipopolysaccharide (LPS) from the cell wall, following antimicrobial therapy $[14,15]$.

Recent research in the management of systemic infection has concentrated on the development of therapy, such as anti-cytokine strategies, to modulate the immune response to infection and to dampen the inflammatory cascade [16]. Indications from preliminary immunotherapy trials suggest that timing is critical, with improved responses resulting from early therapeutic intervention [13]. The development of methods for the early detection of infection, based on the responses of the immune system itself, would clearly be of great advantage [17]. Many studies have already considered the measurement of pro-inflammatory cytokines such as IL6 [17], TNF $\alpha$, IL-1 $\beta$ [18] and IFN- $\gamma$ [10], as indicators of infection. However, determination of these cytokines may not reflect their true level of cellular activity, as serum concentrations may be below detection [10]. Furthermore, the methods of measurement are difficult and the cytokines are of a very labile nature [10]. Therefore, determination of the cellular response to cytokine release may prove a more successful indicator of the immune response.

The results of this study indicate that measurement of the $\mathrm{Fc} \gamma$ receptor response may be used as an early indicator of infection. The expression of $\mathrm{Fc} \gamma \mathrm{RI}$ and the percentage of monocytes demonstrating upregulation of Fc $\gamma$ RIII, were significantly increased in patients with either systemic or localised infection, compared to patients without evidence of bacterial infection and controls. These results are in agreement with previous studies investigating $\mathrm{Fc} \gamma$ receptor response. In 1993, Guyre et al. also found that Fc $\gamma \mathrm{RI}$ expression was increased on neutrophils and augmented on monocytes in patients with localised group A $\beta$-haemolytic streptococcal pharyngeal infection [10]. In a recent study involving HIV-infected patients, the expression of $\mathrm{Fc} \gamma \mathrm{RI}$ and the percentage of monocytes expressing Fc $\gamma$ RIII were also found to be significantly increased, compared to controls [19]. In a further study involving HIV-infected patients, the level of expression of $\mathrm{Fc} \gamma \mathrm{RI}$ and the percentage of monocytes expressing FcyRIII were not only found to be significantly increased, but were also found to have an association with serious or opportunist infections in these patients (unpublished observations).

The results of the present study also indicate that a similar increase in $\mathrm{Fc} \gamma \mathrm{R}$ expression occurred in 

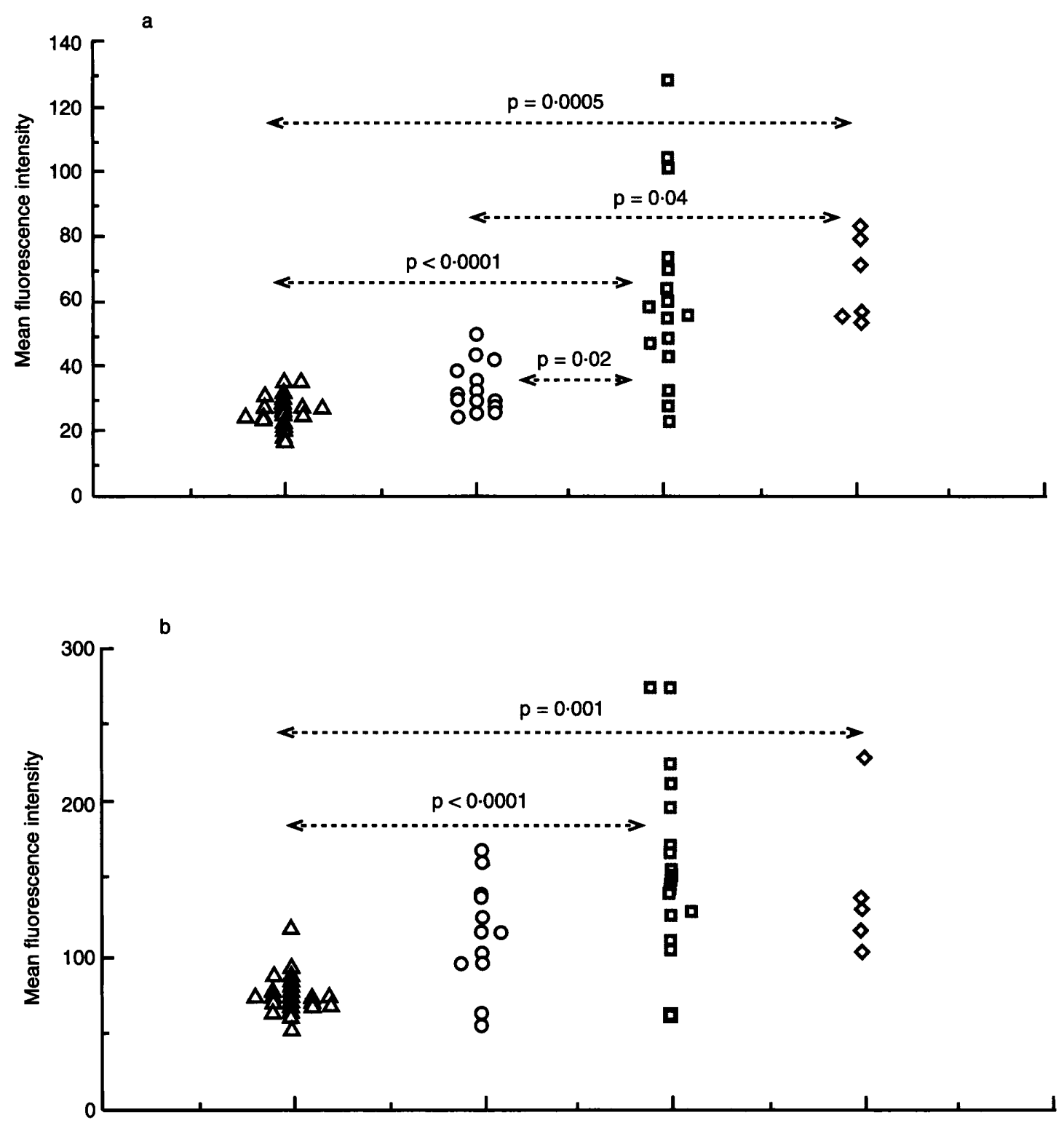

c

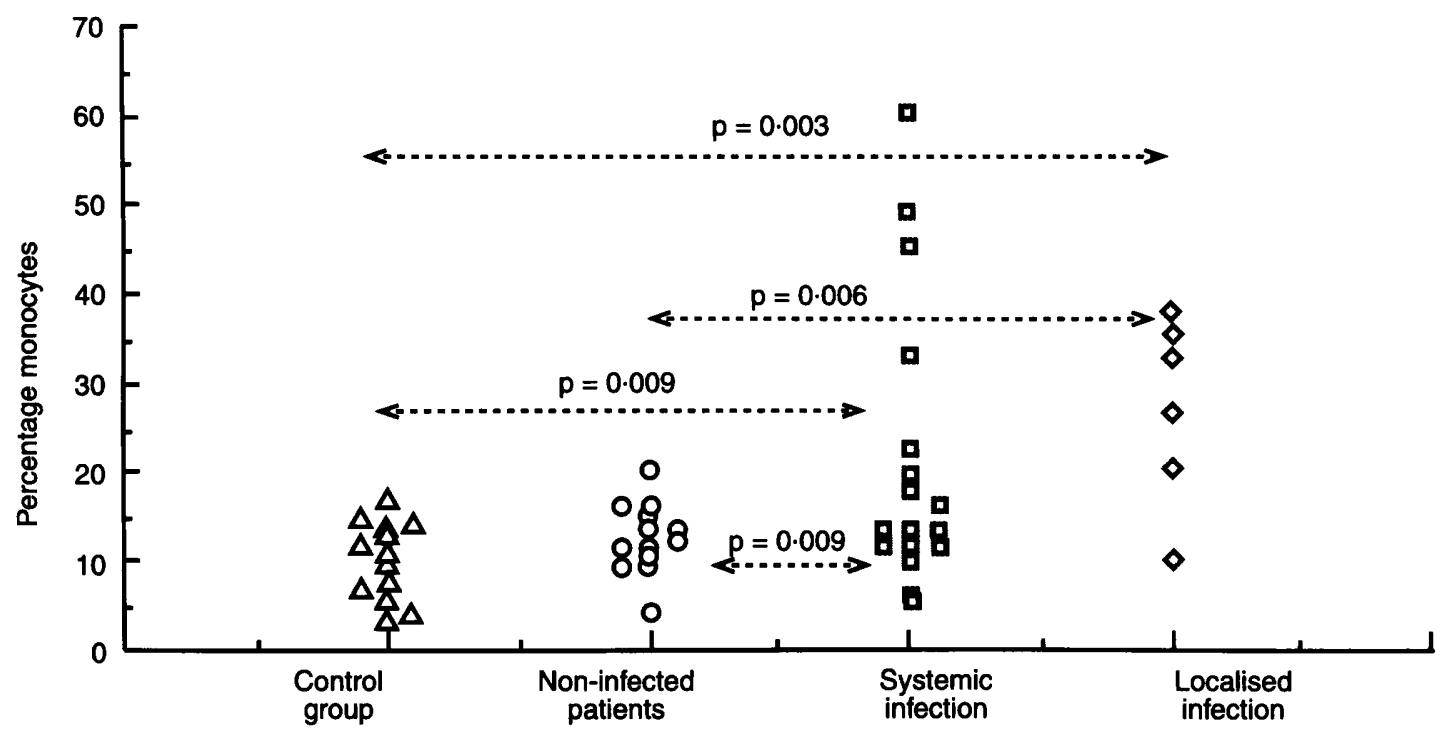

Fig. 1. Fc $\gamma$ receptor expression in patient and control groups: a, mean fluorescence intensity (MFI) values for Fc $\gamma$ RI on neutrophils; b, MFI values for Fc $\gamma$ RI on monocytes; $\mathbf{c}$ percentage of monocytes with increased Fc $\gamma$ RIII expression. Patient groups consisted of non-infected patients, patients with systemic infection and patients with localised bacterial infection; $p$ values are quoted where there is a significant difference $(p<0.05)$ between groups. 

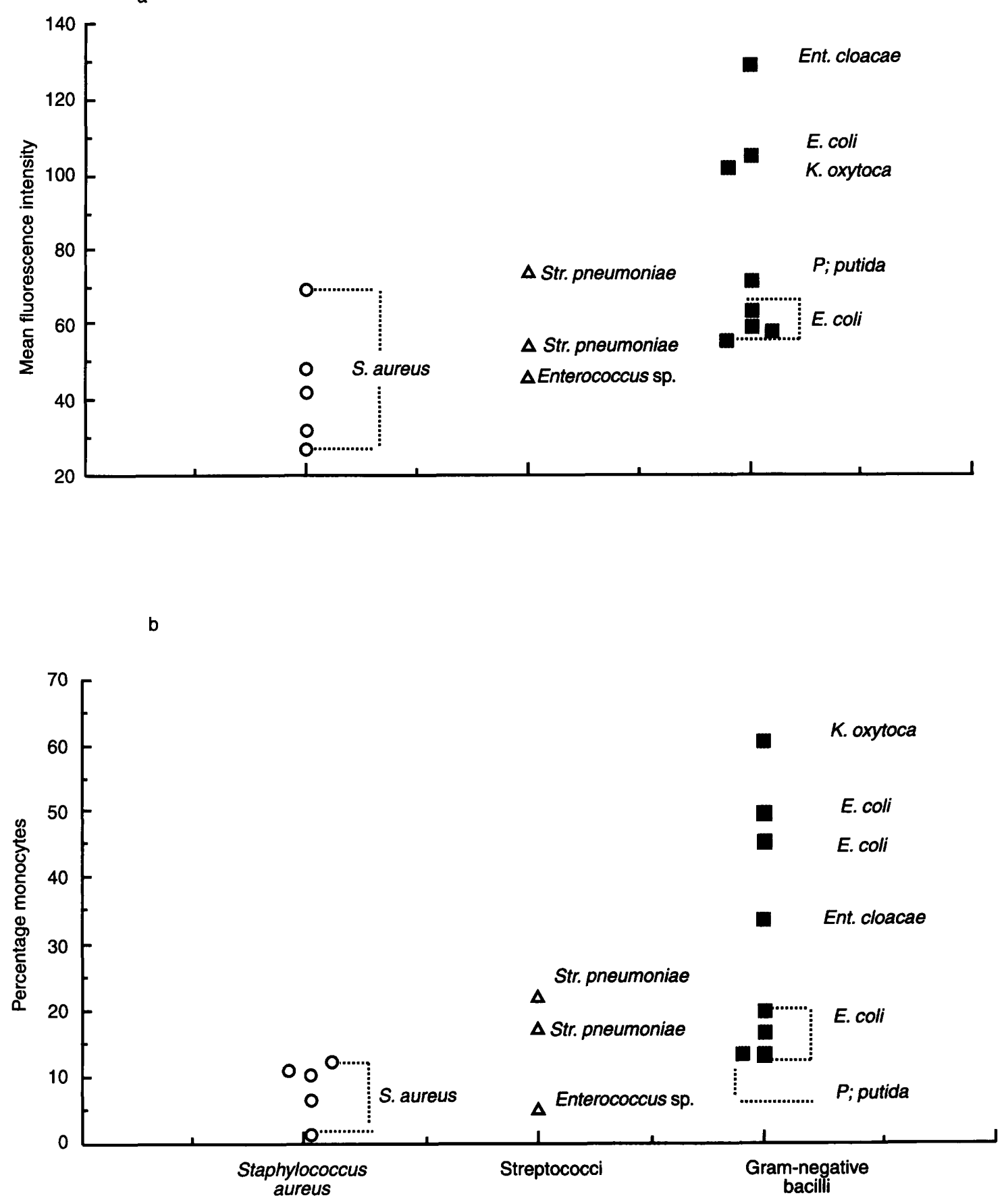

Fig. 2. Fc $\gamma$ receptor expression in patients with systemic infection, for different genera of bacteria: a, mean fluorescence intensity values for Fc $\gamma$ RI on neutrophils; b, percentage of monocytes with increased expression of Fc $\gamma$ RIII.

patients with either localised or systemic infection. However, increased $\mathrm{Fc} \gamma \mathrm{R}$ expression may not occur in all bacterial infections. In a study involving streptococcal infection, Guyre et al. [10] suggested that Fc $\gamma$ RI was selectively increased in some bacterial infections. They found that Fc $\gamma \mathrm{RI}$ expression was upregulated in a small number of patients with pyelonephritis compared to patients with gram-negative urinary tract infections [10]. This might suggest that in localised infection the level of Fc $\gamma \mathrm{R}$ expression will depend on the level of systemic involvement. In the present study, the localised infections examined were of a severe nature, involving gram-negative lower respiratory tract pathogens, and, as a result, may have been sufficient to activate the entire circulating pool of phagocytes. Therefore, these results may indicate that, whilst the measurement of $\mathrm{Fc} \gamma \mathrm{R}$ expression may not distinguish the site of infection, be it bloodborne or localised, measurement could help to indicate the type of infection, in terms of the extent of systemic involvement.

Furthermore, in preliminary studies performed on a patient with gram-negative systemic infection, the Fc $\gamma \mathrm{R}$ response appeared to parallel the course of infection (unpublished data). In the initial period of 
infection the $F c \gamma R$ expression was significantly increased. With resolution of infection, after administration of suitable antimicrobial therapy, the levels returned to control values, suggesting that the measurement of $F c \gamma R$ responses could also be of prognostic value.

The results of the present study also indicate that certain types of bacteria can elicit a higher Fc $\gamma$ receptor response than others. In patients with septicaemia, infections caused by gram-negative bacilli such as $K$. oxytoca, Ent. cloacae and E. coli induced a higher increase in $\mathrm{Fc} \gamma$ receptor expression than either streptococci or staphylococci. In turn, systemic infections caused by streptococci such as Str. pneumoniae, induced a higher response than infections involving $S$. aureus. Similarly, in localised infection, gram-negative bacteria also induced high levels of Fc $\gamma \mathrm{R}$ expression, but to a lesser degree than gramnegative isolates in systemic infection. These results suggest that the measurement of the level of $F c \gamma R$ expression could be used to indicate the type of pathogen involved.

Increased $F c \gamma R$ expression is thought to result from the release of IFN- $\gamma$ from activated $T$ cells after antigenic challenge $[8,9]$. Therefore, during infection, the level of $F c \gamma R$ expression will depend on the ability of the organism to induce IFN- $\gamma$ release. In the case of gram-negative bacilli, bacterial LPS interacts with LPS-binding protein, through the CD14 glycoprotein, to bring about release of proinflammatory cytokines with subsequent T-cell activation [13]. However, recent studies have indicated that LPS can act directly on natural killer cells, to produce an additional source of IFN- $\gamma$ [20]. Taken together this could explain the high level of $F c \gamma R$ expression observed in gram-negative infection in this study. In the case of infection caused by grampositive organisms such as streptococci, increased levels of IFN- $\gamma$ could result from the action of superantigens, e.g., exotoxins and certain $M$ proteins. By circumventing the normal rules of antigen presentation and $\mathrm{T}$-cell recognition, these antigens can stimulate large numbers of $T$ lymphocytes and thus amplify the release of cytokines [21]. Studies of bacteria-induced $F c \gamma R$ expression could further our understanding of the inflammatory response in infection.

The results of this preliminary study suggest that the measurement of $\mathrm{Fc} \gamma \mathrm{R}$ expression could be used as an early indicator of infection. Quantification of the $\mathrm{Fc} \gamma \mathrm{R}$ response might also help to indicate the type or severity of infection and even the organism involved. Furthermore, as the method of determination of Fc $\gamma R$ is both rapid and simple to perform, this test could be used easily in conjunction with standard culture technique and could serve as a useful tool in the treatment of infection.
We thank the following members of the medical staff in the Microbiology Department, St James's Hospital, for providing clinical details on the patients in this study: Drs E. Mulvihill, B. O'Connell, B. Crowley and E. McCarthy. We also thank Ms Anne Forde for her statistical assistance.

\section{References}

1. Parillo JE. Pathogenic mechanisms of septic shock. Mechanisms of disease. $N$ Engl J Med 1993; 328: 1471-1477.

2. Banerjee DK, Hilson GR. Humoral immune response in infection. Curr Opin Infect Dis 1991; 4: 325-331.

3. Unkeless JC. Function and heterogeneity of human Fc receptors for immunoglobulin G. $J$ Clin Invest 1989; 83: 355-361.

4. Fridman WH, Bonnerot C, Daeron M, Amigorena S, Teillaud JL, Sautes C. Structural bases of Fc $\gamma$ receptor functions. Immunol Rev 1992; 125: 49-76.

5. Huizinga TWJ, Roos D, von dem Borne AEGK. Neutrophil Fc $\gamma$ receptors: a two-way bridge in the immune system. Blood 1990; 75: 1211-1214.

6. van de Winkel JGJ, Capel PJA. Human IgG Fc receptor heterogeneity: molecular aspects and clinical implications Immunol Today 1993; 14: 215-221.

7. Simms HH, Frank MM, Quinn TC, Holland S, Gaither TA. Studies on phagocytes in patients with acute bacterial infections. $J$ Clin Invest $1989 ; 83$ : 252-260.

8. Guyre PM, Morganelli PM, Miller R. Recombinant immune interferon increases immunoglobulin $G$ Fc receptors on cultured mononuclear phagocytes. J Clin Invest 1983; 72: 393-397.

9. Petroni KC, Shen L, Guyre PM. Modulation of human polymorphonuclear leukocyte IgG Fc receptors and $\mathrm{Fc}$ receptor-mediated functions by IFN- $\gamma$ and glucocorticoids. $J$ Immunol 1988; 140: 3467-3472.

10. Guyre PM, Campbell AS, Kniffin WD, Fanger MW. Monocytes and polymorphonuclear neutrophils of patients with streptococcal pharyngitis express increased numbers of Type 1 IgG Fc receptors. J Clin Invest 1990; 86: 1892-1896.

11. Stevens DL. Pathogenesis and immune response. Curr Opin Infect Dis 1992; 5: 345-347.

12. Vincent J-L. Newer therapies for septicemia. Curr Opin Infect Dis 1992; 5: 637-641.

13. Stevens D, Bryant AE, Hackett SP. Sepsis syndromes and toxic shock syndromes: concepts in pathogenesis and a perspective of future treatment strategies. Curr Opin Infect Dis 1993; 6: 374-383.

14. Nys M, Damas P, Joassin L, Lamy M. Sequential anti-core glycolipid immunoglobulin antibody activities in patients with and without septic shock and their relation to outcome. Ann Surg 1993; 217: 300-306.

15. Rapson NT. Biological and clinical effects of bacterial lipopolysaccharide. Curr Opin Infect Dis 1988; 1: 919-925.

16. Evans TJ, Cohen J. Immunotherapy of sepsis. $J$ Med Microbiol 1993; 38: 237-239.

17. Sague C, Bland L, Arduino $M$, Mc Allister S, Jarvis W. Utility of serum interleukin-6 (IL-6) levels in differentiating bloodstream infection positive and negative sepsis episodes in neonatal intensive care unit patients. 33rd Interscience Conference on Antimicrobials Agents and Chemotherapy, 1993. Abstract no. 705, p. 248.

18. Brauner A, Wretlind B, Hylander B. Tumor necrosis factor (TNF $\alpha)$ interleukin-1 $\beta$ (IL-1 $\beta$ ) and interleukin-1 receptor antagonist (IL-1ra) in patients on continuous ambulatory dialysis (CAPD) with peritonitis. 33rd Interscience Conference on Antimicrobial Agents and Chemotherapy, 1993. Abstract no. 707, p. 248.

19. Capsoni F, Minonzio F, Ongari AM et al. Fc receptors expression and function in mononuclear phagocytes from AIDS patients: modulation by IFN $\gamma$. Scand $J$ Immunol 1994; 39: $45-50$.

20. Bancroft GJ, Bosma MJ, Bosma GC, Unanue ER. Regulation of macrophage Ia expression in mice with severe combined immunodeficiency: induction of Ia expression by a $\mathrm{T}$ cellindependent mechanism. $J$ Immunol 1986; 137: 4-9.

21. Kotb M. Role of superantigens in the pathogenesis of infectious diseases and their sequelae. Curr Opin Infect Dis 1992; 5: $\quad 364-374$. 